\title{
Novel Polymerization of THF Introducing Fluoroformyl Group as a Polymer End Group Utilizing the Reaction between HFPO and THF for the Initiation - The Effect of $\mathrm{CuF}_{2} /\left(\mathrm{C}_{2} \mathrm{H}_{5}\right)_{3} \mathrm{Al}$ as a Catalyst
}

\author{
Yasuyuki Masubuchi, Katuya Maeyama, Noriyuki YoneZawa, and Tokio Hagiwara*,† \\ Tokyo University of Agriculture \& Technology, Koganei, Tokyo 184-8588, Japan \\ *Saitama Institute of Technology, 1690 Fusaiji Okabe, Saitama 369-0293, Japan
}

(Received September 30, 2002; Accepted December 26, 2003)

\begin{abstract}
Novel poly(THF) with fluoroformyl group at a polymer end was prepared by the reaction of HFPO and THF. Using $\mathrm{CuF}_{2} /\left(\mathrm{C}_{2} \mathrm{H}_{5}\right)_{3} \mathrm{Al}$ as a catalyst fairly higher polymer yields were obtained compared with no catalyst polymerization. The end group of obtained polymer was easily able to be modified to hydroxy or methoxy group. KEY WORDS Hexafluoropropylene Oxide / THF / $\mathrm{CuF}_{2} /\left(\mathrm{C}_{2} \mathrm{H}_{5}\right)_{3} \mathrm{Al} /$
\end{abstract}

Hexafluoropropylene oxide (HFPO) is one of important source chemicals for the development of fluorine containing polymeric materials. HFPO has not given high polymer but only oligomer due to its low reactivity with ionic initiators. ${ }^{1}$ HFPO oligomers are, however, widely utilized as thermally stable materials such as lubricants in various fields. Study on the reactivity of HFPO, - especially on its nature in the polymerization reaction - is thus a key for the development of new fluorine containing materials.

A few reports on the polymerization of cyclic ethers in the system consisting of HFPO and cyclic ether have been found only for ethylene oxide ${ }^{2}$ and $\mathrm{THF}^{3}$ In the previous works, ${ }^{4,5}$ we found novel initiation reaction for the ring-opening polymerization of cyclic ethers in which HFPO was involved. Initiation, propagation, and termination reaction of ring-opening polymerization of cyclic ether initiated with the reaction between HFPO and the cyclic ether were investigated in detail using 3, 4, 5 and 6 menbered cyclic ethers, such as propylene oxide, oxetane, THF and THP. ${ }^{4,5}$ In that study, it was revealed that propylene oxide which has a substituent at 2-position showed different reactivity to HFPO from THF having no substituent, and that thus the polymerized products from the PO/HFPO system were the different from those from THF/HFPO system. ${ }^{4}$

Although HFPO reacts with THF without a catalyst and the resulting product initiates the polymerization of THF as described above, THF has been frequently used as a solvent polymerization or oligomerization of HFPO ${ }^{6,7}$ This is because the reaction of HFPO with THF - by-reaction of the polymerization — is negligible compared with the polymerization of THF in the usual polymerization condition. The reaction of HFPO with THF has been, thus, little noted in the studies of ring-opening polymerization of THF as yet. To study and utilize the reaction of HFPO with cyclic ethers would be, however, quite important and interesting for the synthesis of fluorine containing polymeric materials having polyether structure as a soft segment, especially for modification or functionalization of the polymer ends possessing fluorine atoms, because this reaction could introduce the modificable polymer end at the initial end of polyether. The polymers with fluorine containing modificable polymer end can be expected to prepare new materials for contact lens ${ }^{8}$ and for medical or dental use. ${ }^{9}$ In this paper, we describe that ring-opening polymerization of THF initiated by the reaction of HFPO with THF with $\mathrm{CuF}_{2}$ / $\left(\mathrm{C}_{2} \mathrm{H}_{5}\right)_{3} \mathrm{Al}$ catalyst that was developed by our group. $\mathrm{CuF}_{2} /\left(\mathrm{C}_{2} \mathrm{H}_{5}\right)_{3} \mathrm{Al}$ catalyst involving copper as a 'soft' metal and organoalminum with acidic nature can be expected to enhance the ring-opening polymerization reaction of THF in the system consisting of THF and HFPO.

\section{EXPERIMENTAL}

All experiments related to the polymerization reaction were carried out under nitrogen or argon atmosphere to exclude oxygen and moisture.

\section{Reagents}

$\mathrm{CuF}_{2}$ were used as received. Hexafluoropropylene oxide was dried purified by distillation by calcium sulfate and molecular sieves $4 \mathrm{~A}$ just before use. $\left(\mathrm{C}_{2} \mathrm{H}_{5}\right)_{3} \mathrm{Al}$ was purified by distillation under reduced pressure. Tetrahydrofuran (THF) were dried by refluxing over calcium hydride and potassium and then distilled before use.

${ }^{\dagger}$ To whom correspondence should be addressed (Fax: +81-48-585-6004, E-mail: hagiwara@ @it.ac.jp). 
Table I. Polymerization of THF initiated by the reaction of THF with HFPO

\begin{tabular}{|c|c|c|c|c|c|c|c|}
\hline Run & Cat. & {$[\mathrm{HFPO}] /[\mathrm{THF}]$} & $\begin{array}{c}\text { Temp. } \\
\left({ }^{\circ} \mathrm{C}\right)\end{array}$ & $\begin{array}{l}\text { Time } \\
\text { (h) }\end{array}$ & $\begin{array}{l}\text { Yield } \\
(\%)^{\mathrm{b}}\end{array}$ & $\begin{array}{c}\bar{M}_{\mathrm{n}} \\
\times 10^{-3}\end{array}$ & $\bar{M}_{\mathrm{w}} / \bar{M}_{\mathrm{n}}$ \\
\hline 1 & $\mathrm{CuF}_{2} /\left(\mathrm{C}_{2} \mathrm{H}_{5}\right)_{3} \mathrm{Al}$ & $7 / 93$ & -30 & 24 & 14.7 & 8.0 & 1.4 \\
\hline 2 & & & 20 & 6 & 56.1 & 4.7 & 1.5 \\
\hline 3 & & & 20 & 24 & 44.5 & 4.0 & 1.7 \\
\hline 4 & & & 50 & 24 & 57.8 & 0.6 & 1.4 \\
\hline 5 & & $17 / 83$ & 20 & 24 & 44.0 & 2.5 & 1.9 \\
\hline 6 & & $43 / 57$ & 20 & 24 & 32.8 & 1.0 & 1.4 \\
\hline 7 & None & $7 / 93$ & -30 & 24 & - & - & - \\
\hline 8 & & & 20 & 24 & 37.0 & 1.1 & 1.3 \\
\hline 9 & & & 50 & 24 & 34.1 & 0.5 & 1.4 \\
\hline 10 & & $14 / 86$ & 20 & 24 & 42.0 & 1.1 & 1.4 \\
\hline 11 & & $43 / 57$ & 20 & 24 & 42.0 & 0.6 & 1.4 \\
\hline
\end{tabular}

aPolymn. time: 24 h., [monomer] $/[$ catalyst $]=20 / 1$, without solvent. ${ }^{b}$ Yield $(\%)=$ (polymer obtained, g)/ (total amount of monomer fed, g) $\times 100$.

\section{Polymerization}

Polymerization was carried out under dry nitrogen in a sealed ampoule which was carefully flame dried. In the ampoule, $\mathrm{CuF}_{2} /\left(\mathrm{C}_{2} \mathrm{H}_{5}\right)_{3} \mathrm{Al}$ system was prepared first by addition of $\left(\mathrm{C}_{2} \mathrm{H}_{5}\right)_{3} \mathrm{Al}$ to $\mathrm{CuF}_{2}$. The reaction mixture prepared was a homogeneous dark solution. Then THF and HFPO were introduced there and the ampoule was fused and sealed. The reaction mixture was poured into a large amount of water. The resulting precipitate which was contaminated with copper, was extracted with chloroform, and washed three times with diluted $\mathrm{HNO}_{3}$ and then water, respectively. The extracted solution was concentrated by evaporation and dried in vacuo. For the polymerization of catalyst free system, the polymer was isolated by precipitation from a large amount water, extracted with chloroform. The extracted solution was washed with water and then dried over magnesium sulfate anhydride. After filtration, chloroform was removed and dried under reduced pressure.

\section{Measurement}

${ }^{1} \mathrm{H},{ }^{13} \mathrm{C}$ and ${ }^{19} \mathrm{~F}$ NMR spectra were recorded on a JEOL JNM-ECP 500 NMR spectrometer using $\mathrm{CDCl}_{3}$ as a solvent. ${ }^{13} \mathrm{C}$ NMR spectrum was measured under proton irradiated condition. Chemical shifts are given in $\mathrm{ppm}$ with plus value for downfield shift ( $\delta$-scale) from tetramethylsilane (TMS) for ${ }^{1} \mathrm{H}$ and ${ }^{13} \mathrm{CNMR}$ as well as trichlorofluoromethane for ${ }^{19} \mathrm{~F}$ NMR. Gel permeation chromatography (GPC) was conducted with RI- and UV-detectors with TSK gel GMHXL $\times$ 2-Shodex KF-802 column. The molecular weight calibration was carried out with polystyrene standard samples. The molecular weight estimated from GPC was in fairly good agreement with that from NMR data. Thermogravimettic analysis (TGA) was carried out with TA Instruments TGA 51 thermogravimeter (sample weight $c a .10 \mathrm{mg}$ ), with Thermal Analyst 2000
(TA Instruments), under argon atmosphere with heating rates of $10 \mathrm{k} / \mathrm{min}$.

\section{RESULTS AND DISCUSSION}

\section{Polymerization}

Results of the polymerization of THF in the system consisting of HFPO and THF with $\mathrm{CuF}_{2} /\left(\mathrm{C}_{2} \mathrm{H}_{5}\right)_{3} \mathrm{Al}$ and without the catalyst are shown in Table I. When initial molar ratio of HFPO/THF is $7 / 93$, polymer was obtained in $37 \%$ yield for no catalyst system at $20^{\circ} \mathrm{C}, 24 \mathrm{~h}$. For the polymerization system with $\mathrm{CuF}_{2} /\left(\mathrm{C}_{2} \mathrm{H}_{5}\right)_{3} \mathrm{Al}$, the yield was much improved to be, $56.1 \%$ for $6 \mathrm{~h}$ and $44.5 \%$ for $24 \mathrm{~h}$ under the same polymerization condition. These results suggest that $\mathrm{CuF}_{2} /\left(\mathrm{C}_{2} \mathrm{H}_{5}\right)_{3} \mathrm{Al}$ works as a catalyst which promotes the polymerization of THF initiated by the reaction of HFPO with THF in the polymerization system consisting of HFPO and THF.

The temperature effect on the polymer yield is very interesting. For the usual THF polymerization systems, high temperature is not so preferable to obtaining high polymer yield. ${ }^{10}$ In the polymerization of $[\mathrm{HFPO}] /[\mathrm{THF}]=7 / 93$, the polymer yields at $50^{\circ} \mathrm{C}$ were slightly lowered compared with those at $20^{\circ} \mathrm{C}$ and molecular weights of the obtained polymer were considerably lowered from $1.1 \times 10^{3}$ (at $20^{\circ} \mathrm{C}$ ) to $0.5 \times 10^{3}$ (at $50^{\circ} \mathrm{C}$ ) for the polymerization without $\mathrm{CuF}_{2} /\left(\mathrm{C}_{2} \mathrm{H}_{5}\right)_{3} \mathrm{Al}$. For the polymerization involving $\mathrm{CuF}_{2} /\left(\mathrm{C}_{2} \mathrm{H}_{5}\right)_{3} \mathrm{Al}$ the decline of the polymer yield is not observed, though molecular weights of the obtained polymer were also significantly decreased from $4.0 \times 10^{3}$ (at $20^{\circ} \mathrm{C}$ ) to $0.6 \times 10^{3}$ (at $50^{\circ} \mathrm{C}$ ). Very low temperature is also unfavored for the polymerization. At $-30^{\circ} \mathrm{C}$, no polymer was obtained without using the catalyst while $14.7 \%$ yield was obtained in the polymerization system with $\mathrm{CuF}_{2} /\left(\mathrm{C}_{2} \mathrm{H}_{5}\right)_{3} \mathrm{Al}$. Significant influence of the initial molar ratio of HFPO/THF 
on the polymer yields was not observed for no catalyst system (yield: $c a .40 \%$. at $20^{\circ} \mathrm{C}$ ), but observed on the molecular weight of polymer. In the case of [HFPO]/ $[\mathrm{THF}]=43 / 57$, the molecular weight of the polymer was about half as much as those in the other cases. On the other hand, for the system with $\mathrm{CuF}_{2} /\left(\mathrm{C}_{2} \mathrm{H}_{5}\right)_{3} \mathrm{Al}$, the influence of the initial molar ratio of HFPO/THF was observed on both yield and molecular weight. When initial molar ratio of HFPO/THF was close to equal, both yield and molecular weight were lowered compared with those obtained from the polymerization in the lower initial molar ratio.

\section{Characterization of Obtained Polymer and Mechanis- tic Aspect of Polymerization}

Figure 1a shows the typical ${ }^{13} \mathrm{C}$ NMR spectrum of the obtained polymer (run 3 in Table I). The signals at 25 and $70 \mathrm{ppm}$ can be assigned to metylene carbons of THF units. The absorptions observed at 100-105 and $115-125 \mathrm{ppm}$ are assignable to fluoromethine and trifluoromethyl carbons of HFPO units, respectively. The doublet signals observed at $80-90 \mathrm{ppm}$ are derived from monofluoromethyl carbon and the singlet signal at $160 \mathrm{ppm}$ is an absorption of carbonyl carbon. The interesting fact that no signal from di-

\section{(A)}
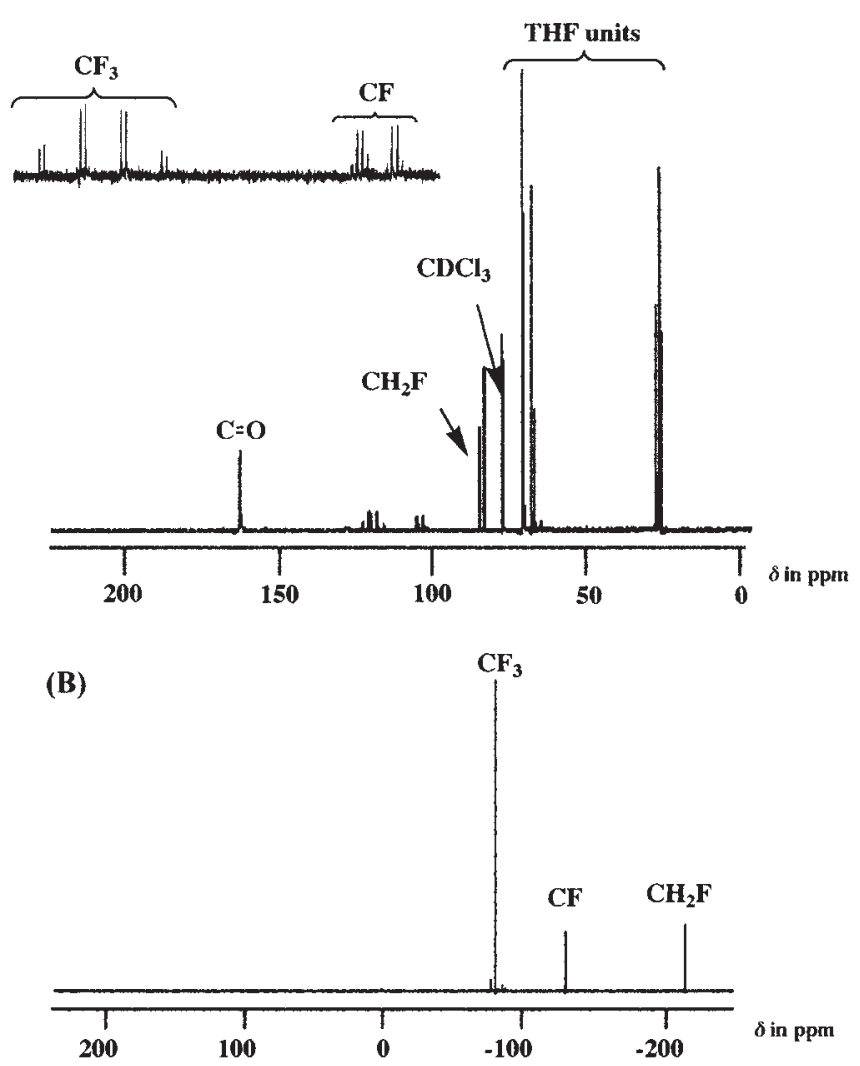

Figure 1. ${ }^{13} \mathrm{C}$ NMR (A) and ${ }^{19} \mathrm{~F} \mathrm{NMR}$ (B) spectra of the polymer obtained with the reaction of HFPO and THF (run 8 in Table I). fluoromethylene carbon being originally in HFPO is observed should be noted. Figure $1 \mathrm{~b}$ shows the ${ }^{19}$ F NMR spectrum of the same sample. The peaks at -78 and $-130 \mathrm{ppm}$ are able to assigned to trifluoromethyl and fluoromethylene groups of HFPO units, respectively. The signal at $-218 \mathrm{ppm}$ can be assigned to monofluoromethyl group. As well as in the ${ }^{13}$ C NMR spectrum, the signals from difluoromethylene group are not found. These NMR results indicate that difluoromethylene group in HFPO unit disappears in the process of polymerization. This implies that this polymerization reaction does not proceeds through usual ring opening mechanism. The disappearance of difluoromethylene group in HFPO unit can be explained by the specific initiation mechanism of this polymerization as shown in Scheme 1: the initiation reaction takes place, at first, by the reaction of HFPO which can coordinate to $\mathrm{CuF}_{2} /\left(\mathrm{C}_{2} \mathrm{H}_{5}\right)_{3} \mathrm{Al}$ complex with THF, and the initiating species produced releases fluoride anion ((I), (II) in Scheme 1).

In this reaction process, $\mathrm{CuF}_{2}$ and $\left(\mathrm{C}_{2} \mathrm{H}_{5}\right)_{3} \mathrm{Al}$ is considered to form an ate complex type species, which would work as a catalyst. As shown in Table I, the polymerization system involving $\mathrm{CuF}_{2} /$ $\left(\mathrm{C}_{2} \mathrm{H}_{5}\right)_{3} \mathrm{Al}$ gives poly(THF) with a fluoroformyl end group at $-30^{\circ} \mathrm{C}$, while no polymer is obtained without $\mathrm{CuF}_{2} /\left(\mathrm{C}_{2} \mathrm{H}_{5}\right)_{3} \mathrm{Al}$. Furthermore, the acceleration of the polymerization reaction is observed for the polymerization system with $\mathrm{CuF}_{2} /\left(\mathrm{C}_{2} \mathrm{H}_{5}\right)_{3} \mathrm{Al}$ in the low initial molar ratio of HFPO to THF ([HFPO]/ $[\mathrm{THF}]=7 / 93$ ) at $20^{\circ} \mathrm{C}$ (run 2 , run 3 and run 8). In the polymerization at higher ratios of [HFPO]/ [THF], significant difference of the polymerization behavior is not observed for polymerization systems - with or without $\mathrm{CuF}_{2} /\left(\mathrm{C}_{2} \mathrm{H}_{5}\right)_{3} \mathrm{Al}$. These results apparently suggest that $\mathrm{CuF}_{2} /\left(\mathrm{C}_{2} \mathrm{H}_{5}\right)_{3} \mathrm{Al}$ participates in the initiation process of the polymerization in which HFPO would play an important role rather than propagation in which HFPO does not take part.

The propagation reaction of the polymerization is considered to proceed through oxonium ion mechanism with the $\mathrm{CuF}_{2} /\left(\mathrm{C}_{2} \mathrm{H}_{5}\right)_{3} \mathrm{Al}$ complex ((III) and (IV) in Scheme 1).

According to Scheme 1, the polymer produced should have fluoroformyl (CFO) group at one end of the polymer chain due to the initiation reaction. On the NMR spectra shown in Figure 1, however, the signal assignable to CFO group could not be found while ordinaly carbonyl group is detected in ${ }^{13} \mathrm{C}$ NMR. This is due to hydrolysis of $\mathrm{CFO}$ group to $-\mathrm{OH}$ group by treatment with water in the isolation process of the polymer (see Experimental section). In fact, when obtained polymer was dipped into methanol before treatment with water, the end group of the polymer was changed to $-\mathrm{OCH}_{3}$ group. This was confirmed by 


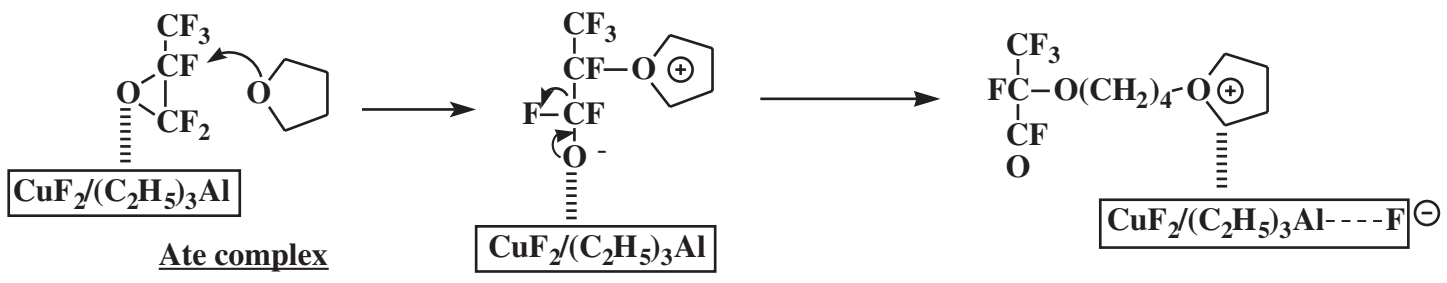

(I)

(II)

(III)

Initiation Step

Propagetion Step

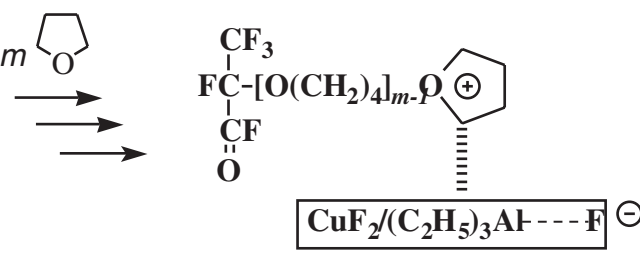

(IV)

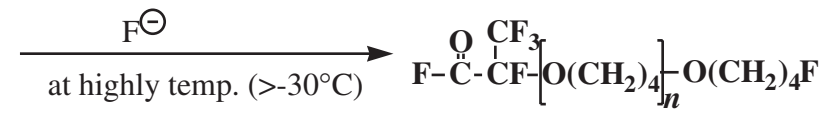

(V)

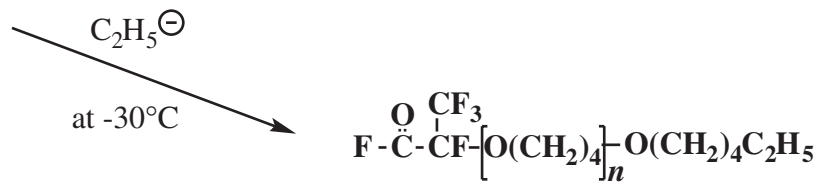

(VI)

Termination Step

Scheme 1.

NMR analysis.

Figure 2 shows the ${ }^{1} \mathrm{HNMR}$ spectra of the polymers obtained from the polymerization above room temperature treated with water (a) and methanol (b). In Figure 2a, the absorption of hydroxy group is observed around $10.3 \mathrm{ppm}$ as a broadened signal. For the methanolysis polymer, the signal of methoxy group is clearly observed at $3.91 \mathrm{ppm}$ in Figure $2 \mathrm{~b}$, and the intensity ratio of the $-\mathrm{OCH}_{3}$ signal which was introduced by methanolysis of -CFO generated in the initiation step to $-\mathrm{CH}_{2} \mathrm{~F}$ given as a termination end by the termination with fluoride anion is $3: 2$. This result indicates that the all polymerization reaction initiated with the species from HFPO is terminated with only fluoride anion in the polymerization system with $\mathrm{CuF}_{2} /\left(\mathrm{C}_{2} \mathrm{H}_{5}\right)_{3} \mathrm{Al}$ above room temperature $((\mathrm{V})$ in Scheme 1).

On the other hand, in the polymerization system at $-30^{\circ} \mathrm{C}$, the signal intensity ratio of $-\mathrm{OCH}_{3}$ to $\mathrm{CH}_{2} \mathrm{~F}$ is 9:2 for the polymer obtained after methanolysis as illustrated in Figure 3a. This polymer is confirmed to have the HFPO initiating unit by ${ }^{19}$ F NMR spectrum (Figure 3b) as well as the other resulting polymers, i.e. this polymer also has fluoroformyl group at initiating end quauntitatively. This means that termination reaction other than that with fluoride anion would occur in the polymerization reaction at $-30{ }^{\circ} \mathrm{C}$ with $\mathrm{CuF}_{2} /\left(\mathrm{C}_{2} \mathrm{H}_{5}\right)_{3} \mathrm{Al}$ complex: for instance, termination with ethyl group which can migrate from triethylaluminium ((VI) in Scheme 1). In fact, the signals assignable to ethyl group are observed on Figure $3 \mathrm{a}$ as triplet $(1.34 \mathrm{ppm})$ and quartet $(1.48 \mathrm{ppm})$ signals, respectively. The polymerization of THF in the presence of HFPO as an initiating species generator gives the poly(THF) with a fluoroformyl end group for each polymer chain whether $\mathrm{CuF}_{2} /\left(\mathrm{C}_{2} \mathrm{H}_{5}\right)_{3} \mathrm{Al}$ is in the polymerization system or not. As mentioned above, the fact that the fluoroformyl group can be introduced quantitatively at one end of each polymer chain and easily transformed other functional groups by simple post-treatment suggests that the polymer obtained from these polymerization systems consisting of HFPO and THF have versatile potentiality for the reactivity polymeric material.

The polymers which were obtained from different post-treatment showed different thermal property. Figure 4 shows TGA curves of the polymers treated with water (a) and methanol (b). For the TGA curve of the polymer treated with water, thermal decomposition starts around $200^{\circ} \mathrm{C}$. The temperature of $10 \%$ weight loss is $215^{\circ} \mathrm{C}$, which is slightly more stable than that of the polymer treated with methanol. This higher thermal property observed in the TGA profile for the polymer with hydroxyl end group may be due to the hydrogen bond formed by hydroxyl groups. 
THF units

(A)

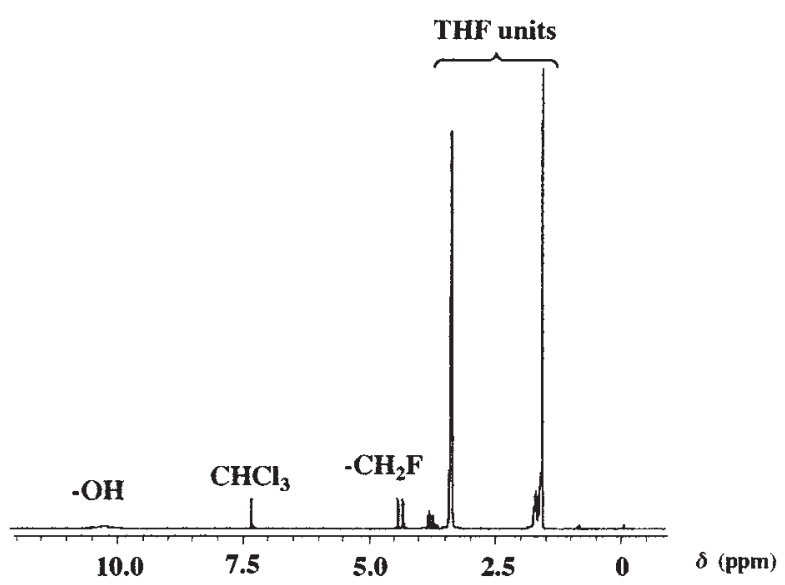

(B)

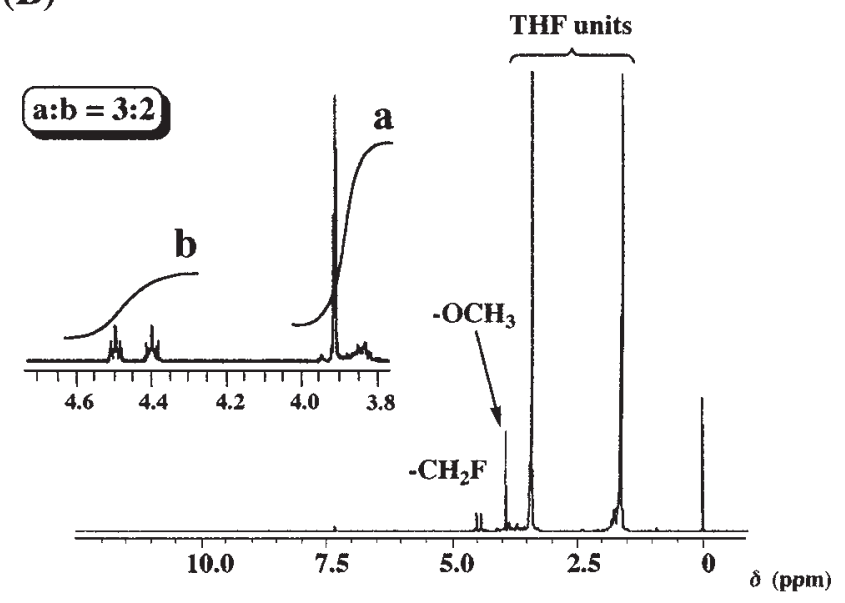

Figure 2. ${ }^{1} \mathrm{H}$ NMR spectrum of the polymer obtained with the reaction of HFPO and THF in the presence of $\mathrm{CuF}_{2} /\left(\mathrm{C}_{2} \mathrm{H}_{5}\right)_{3} \mathrm{Al}$ at $20^{\circ} \mathrm{C}$ treated with water (A) and methanol (B) (run 3 in Table I).

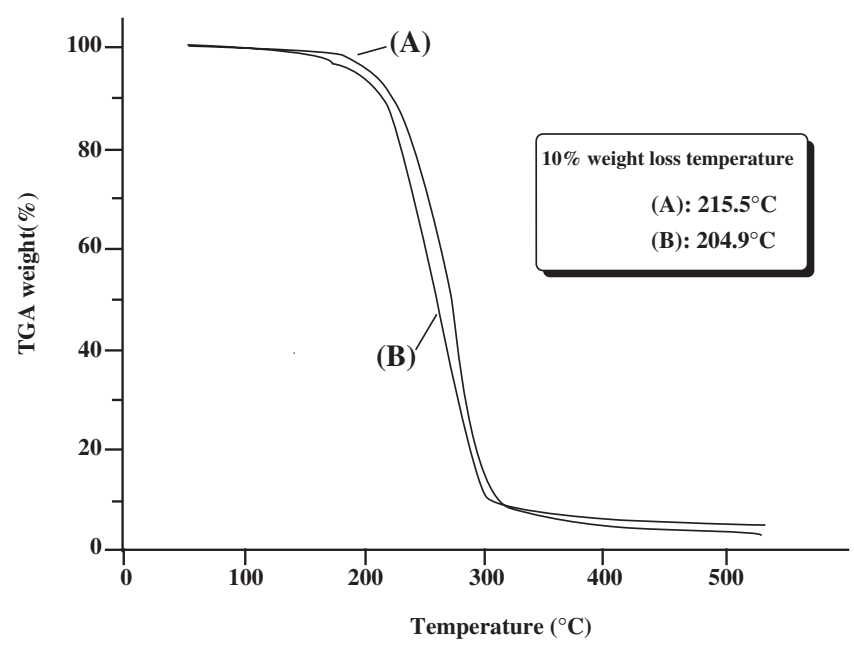

Figure 4. TGA profiles of the water-treated (a) and methanoltreated (b) polymers.
(A)

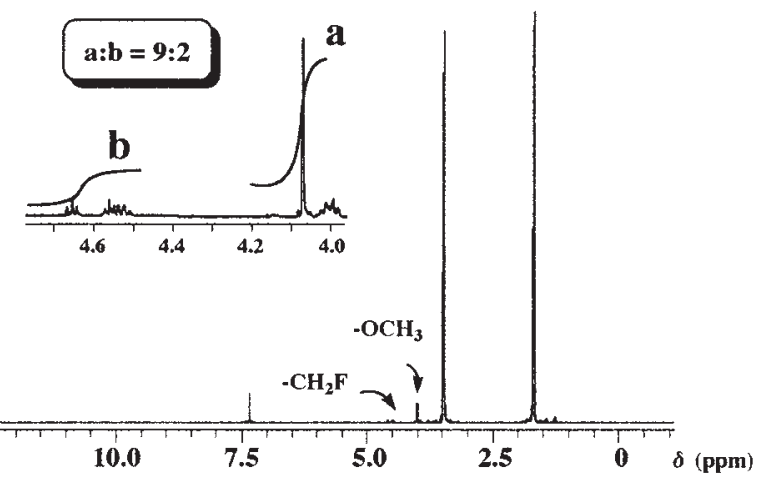

(B)

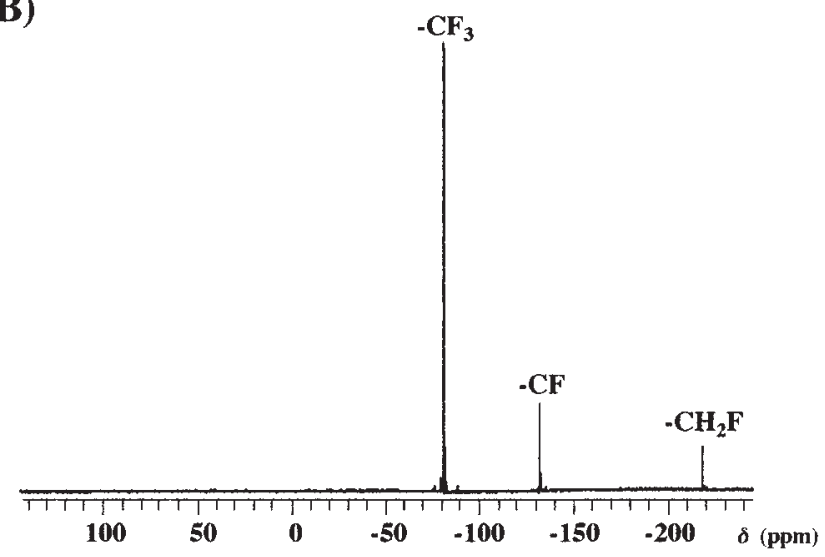

Figure 3. ${ }^{1} \mathrm{H}$ NMR (A) and ${ }^{19} \mathrm{FNMR}$ (B) spectra of the polymer obtained with the reaction of HFPO and THF in the presence of $\mathrm{CuF}_{2} /\left(\mathrm{C}_{2} \mathrm{H}_{5}\right)_{3} \mathrm{Al}$ at $-30{ }^{\circ} \mathrm{C}$ treated with methanol (run 1 in Table I).

\section{REFERENCES}

1. H. S. Eleuterio, J. Macromol. Sci. Chem. A, 6, 1027 (1972).

2. H. Millauer, W. Schwertfeger, and G. Siegemund, Angew. Chem., Int. Ed. Engl., 24, 161 (1985).

3. T. G. Shchibrya, S. P. Krukovski, A. V. Ignatenko, and V. A. Ponomarenko, Vyskomol. soedin., Ser. B, 21, 517 (1979).

4. Y. Masubuchi, T. Hagiwara, Y. Ikegaya, and N. Yonezawa, Nippon Kagaku Kaishi, 3, 365 (2002).

5. Y. Masubuchi, K. Maeyama, N. Yonezawa, and T. Hagiwara, Kobunshi Ronbunshu, 7, 329 (2003).

6. N. Ishikawa, Yuki Gosei Kagaku Kyoukaishi, 35, 131 (1977).

7. G. M. Tyul'ga, V. A. Guvanov, D. S. Rondarev, R. M. Ryazanova, and I. M. Dolgopol'skii, Zh. Org. Khim., 16, 300 (1980).

8. S. Ichinoe, K. Takahashi, and K. Tanaka, Jp, 62-008769, B (1987).

9. E. Masubara, Y. Kodama, Y. Imai, and M. Ueda, Jp, 02-001164, B (1990).

10. M. P. Dreyfuss and P. Dreyfuss, J. Polym. Sci., Part A-1: Polym. Chem., 4, 2179 (1966). 